\title{
Specific Immunotherapy in Advanced Cervical-Uterine Cancer Using Humanized Monoclonal Antibody Nimotuzumab and CIMAvax-EGF ${ }^{\circledR}$ Therapeutic Vaccine
}

\author{
Raiza Ruiz-Lorente1, Sayly Alfonso², Eduardo Santiesteban ${ }^{3}$, Yamilka Sánchez ${ }^{4}$, \\ Kirenia Camacho5, Erasmo Mendoza ${ }^{5}$, Carmen Elena Viada ${ }^{6}$, Ramón Ortiz ${ }^{2}$, \\ Ihosvannys Carreño ${ }^{5}$, Mayelin Troche ${ }^{6}$, Meylan Cepeda ${ }^{6}$, Ana Rosa Vals ${ }^{6}$, Leticia Cabrera ${ }^{6}$, \\ Annia Gorte6, Delmis Batista6, Milagros Domenech6, Yanela Santiesteban', \\ Yuliannis Santiesteban', Daymys Estevez ${ }^{6}$, Jessica García-Viamontes' ${ }^{1}$, \\ Conrado Ramos Mico', Mayra Ramos-Suzarte ${ }^{6 *}$
}

${ }^{1}$ González Coro Hospital, La Habana, Cuba

${ }^{2}$ Celestino Hernández Robau Hospital, Villa Clara, Cuba

${ }^{3}$ Jose Ramón Lopez Tabranes Hospital, Matanzas, Cuba

${ }_{4}^{4}$ III Congreso Hospital, Pinar del Río, Cuba

${ }^{5}$ Faustino Perez Hospital, Matanzas, Cuba

${ }^{6}$ Center of Molecular Immunology, Havana, Cuba

Email: ^mayra@cim.sld.cu

How to cite this paper: Ruiz-Lorente, R., Alfonso, S., Santiesteban, E., Sánchez, Y., Camacho, K., Mendoza, E., Viada, C.E., Ortiz, R., Carreño, I., Troche, M., Cepeda, M., Vals, A.R., Cabrera, L., Gorte, A., Batista, D., Domenech, M., Santiesteban, Y., Santiesteban, Y., Estevez, D., García-Viamontes, J., Mico, C.R. and Ramos-Suzarte, M. (2021) Specific Immunotherapy in Advanced Cervical-Uterine Cancer Using Humanized Monoclonal Antibody Nimotuzumab and CIMAvax-EGF ${ }^{\oplus}$ Therapeutic Vaccine. Journal of Cancer Therapy, 12, 146-156.

https://doi.org/10.4236/jct.2021.123016

Received: February 6, 2021

Accepted: March 22, 2021

Published: March 25, 2021

\begin{abstract}
Cervical uterine cancer represents the fourth most common malignant neoplasm worldwide in the female sex in terms of incidence, principally from epithelial origen. The high expression of EGFR in this tumor leads to the search for therapeutic alternatives. An Expanded Access Clinical Program was carried out in parallel groups, randomized, multicenter and prospective study, to evaluate the survival of patients with advanced cervical carcinoma, without therapeutic alternative, who would be treated with the therapeutic vaccine CIMAvax-EGF ${ }^{\oplus}$, the humanized $\mathrm{mAb}$ nimotuzumab or the combination of both products, which targeted EGF and EGFR respectively. The patients were included between 2008 and 2010 with a more than five years follow-up. The results show that the serious adverse events related to the experimental treatments were $0.9 \% ; 1.1 \%$ and $2.6 \%$ and a median ITT survival of 9.1, 23.5, and 16.3 months for CIMAvax-EGF ${ }^{\circledast}$, nimotuzumab and the combination of both, respectively. Thus fulfilling the hypothesis of safety and efficacy proposed in the investigation was achieved. The three therapeutic regimens achieved overall survival rates greater than $35 \%$ at 60 months, encouraging
\end{abstract}


Copyright $\odot 2021$ by author(s) and Scientific Research Publishing Inc. This work is licensed under the Creative Commons Attribution International License (CC BY 4.0).

http://creativecommons.org/licenses/by/4.0/ results for advanced uterine cervical cancer. A phase III clinical trial is proposed to consolidate these results in a greater number of patients with nimotuzumab as study drug.

\section{Keywords}

CIMAvax-EGF ${ }^{\circledast}$, Nimotuzumab, Survival, Safety, Advanced Cervical Cancer

\section{Introduction}

Cervical uterine cancer represents the fourth malignant neoplasm with the highest incidence in the world in women with 569,847 in 2018. It is also reported as the fourth cause of death from cancer in this sex, being Latin America and the Caribbean a health problem public, with a mortality rate of 7.1/100,000 inhabitants, which is the third in the world [1].

These neoplasms predominantly had epithelial origin, with overexpressing the Epidermal Growth Factor Receptor (EGFR) in a high percentage [2] [3] [4]. The high expression of EGFR in this type of patients leads to the search for therapeutic alternatives against this therapeutic target. Although there are reports of some studies with anti EGFR monoclonal antibodies (mAbs) in this indication, in advanced stages, the results have not been as expected.

The humanized mAb nimotuzumab directed against EGFR has been demonstrated a therapeutic efficacy, with increased survival in patients with persistent, recurrent or advanced-stage cervical cancer used as monotherapy [5] [6]. On the other hand, the CIMAvax EGE vaccine has shown therapeutic efficacy in patients with non-small cell lung tumors (NSCLC) [7], due to its capacity to develop anti EGF antibodies able to reduce circulating EGF concentrations by increasing the antibody titer in treated patients.

The use of both products in the treatment of epithelial uterine cervical cancer using them as monotherapy or in a combination of both could become a therapeutic alternative in advanced disease patients.

\section{Material and Method}

A clinical study classified as Expanded Access Clinical Program (EACP), randomized (by ASAL randomized software 1.2 for windows), multicenter, prospective were conducted, in five hospitals, to evaluate the survival of patients with advanced cervical carcinoma without any therapeutic alternative. The patients, that received the therapeutic cancer vaccine CIMAVAX-EGF ${ }^{\oplus}$, the mAb humanized nimotuzumab or the combination of both were enrolled in three groups, used a random numbers list from April 4th, 2008 to May 17th, 2010. Patients with a diagnosis confirmed by cyto-histological techniques as persistent, recurrent or advanced cervical cancer, who had received all available lines of treatment for its tumor, were taken into account as inclusion criteria, any subject with oncospecific treatment in the moment of inclusion, who gave their in- 
formed consent, with an ECOG $\leq 3$. Patients who were receiving another product under investigation, who had previously received the products under evaluation, with decompensated chronic diseases or general status $>3$, were excluded. The classification of adverse events was according to the common toxicity criteria (CTC) of the National Cancer Institute od United State of America, version $3.0[8]$.

The hypothesis of the study was proposed taking into account the safety and efficacy profile of the treatments. Success was considered if less than $20.0 \%$ of the patients in the treated groups developed related serious adverse events (SAE) and if the patients reach an overall survival advantage of at least three months (after two years of follow-up), with respect to conventional therapy (which at the time of initiation of the study was not longer than seven months).

\subsection{Research Products}

CIMAvax-EGF ${ }^{\varpi}$ vaccine: It is the result of the emulsion of the chemical conjugate of $1 \mathrm{mg}$ of recombinant human EGF (EGF hu-rec) P64k protein from Neisseria Meningitides (P64k rec) with the adjuvant Montanide ISA 51. It was administered intramuscularly, $4 \mathrm{mg}$ of the vaccine per dose, at four immunization points, two higher and two lower. The treatment was planning in two stages: induction with vaccine administration every 14 days, 5 times and Maintenance every 28 days for at less two years.

Nimotuzumab (CIMAher $\left.{ }^{\circledR}\right)$ : Is ahumanized monoclonal antibody, IgG1 subclass. It was administered intravenously, $200 \mathrm{mg}$ per dose over 1 hour. Treatment divided into two stages: Induction stage once a week for 12 consecutive weeks and maintenance stage once every 14 days.

Nimotuzumaband CIMAvax-EGF ${ }^{\circledast}$ vaccine combination: was administered in schedules and doses similar to the two previous groups, on different days to identify the toxicity associated with each type of treatment.

In all cases, treatment continued beyond progression of the disease, until the appearance of unmanageable toxicity or the worsening of the general condition of the patient ECOG > 3.11.

\subsection{Safety}

All randomized patients were evaluable after receiving at least one dose. For the toxicity analysis, the adverse events (AE) presented by the patients in each of the treatment groups were compared according CTCAE version 3 [8].

\subsection{Statistics}

The sample size calculation used binomial distribution for pilot clinical trial of translational therapies, which allows obtaining a value from which an upper limit of toxicity can be estimated [9]. A range of number of patients per treatment group of $44-49$ was established by statistical criterion according to power, assuming that $80 \%$ would be used (49 patients per treatment group taking into 
account $10 \%$ additional). By presetting a maximum tolerable toxicity limit (20.0\%), the sample size can be determined to take into account type I error = 0.05 and a maximum tolerable toxicity ratio $=0.20$. To answer the efficacy hypothesis A bilateral hypothesis assuming type I error alpha $=0.05$, type II error beta $=0.2$, a difference of three months of survival related to the most frequent reference value in the literature reported in the year that the investigation begins (NCCN. 2009, QRTaround 6 months) [10].

\section{Results and Discussion}

The EGFR receptor and its ligands have been a widely studied therapeutic target in tumors of epithelial origin and it is associated as a poor prognostic factor in this disease [11].

In Cuba, in 2008, given the low availability of therapeutic options in the second line of treatment in advanced cervical cancer (recurrent, persistent or metastatic), the use of therapies directed against the available therapeutic EGF-EGFR target was proposed.

In this Expanded Access Clinical Program (EACP), 180 patients with a diagnosis of carcinoma of the cervix of epithelial origin, refractory to all previous therapies due to advanced disease, were included in an expanded clinical study (EACP) distributed in three groups of patients. Randomized 75 patients in the nimotuzumab group, 59 inCIMAvax-EGF ${ }^{\varpi}$ vaccine and 46 in the combination of both products. $74.4 \%$ of the patients were included with an acceptable general state (ECOG 0-1). Squamous cell carcinoma was the predominant histological type (54.4\%). A significant association was observed for the histological subtypes in the three groups analyzed according to the estimate by the $\mathrm{X}^{2}$ test, with a higher proportion of Adenocarcinoma (ADC) in the group treated with the CIMAvax-EGF ${ }^{\circledast}$ vaccine. The rest of the variables were balanced.

Of 1018 AEs described, $45.7 \%$ were related to treatment and of which $12.3 \%$ were classified as serious (Figure 1). Related SAE were $0.9 ; 1.1 \%$ and $2.6 \%$ for CIMAvax-EGF ${ }^{\circledast}$, nimotuzumab and the combination respectively, a proportion lower than the established in the working hypothesis. Therapeutics were well tolerated and proved the possibility to be used as prolonged therapy, beyond progression even when two of them were administered in the same patient as combination therapy. Similar reports have been found in the clinical trials concluded with nimotuzumab and the CIMAvax $\mathrm{EGF}^{\circledast}$ vaccine in other cancer indications, where they have been applied in monotherapy modality or in combination with chemo radiotherapy, for prolonged periods such as head and neck [12] and high-grade glioblastomas of malignancy [13] among others [14]. In a multicenter study in persistent, recurrent, or metastatic cervical cancer by Cetina et al. used nimotuzumab combined with chemotherapy was shown to be well tolerated [5]. The present study, in which added Cimavax egf vaccine into the treatment scheme of nimotuzumab, the toxicity of the treatments was similar.

Survival rates were up to 5 years. ITT and PP analysis was not statistical different however, it is remarkable that for the three therapeutic regimens, overall 


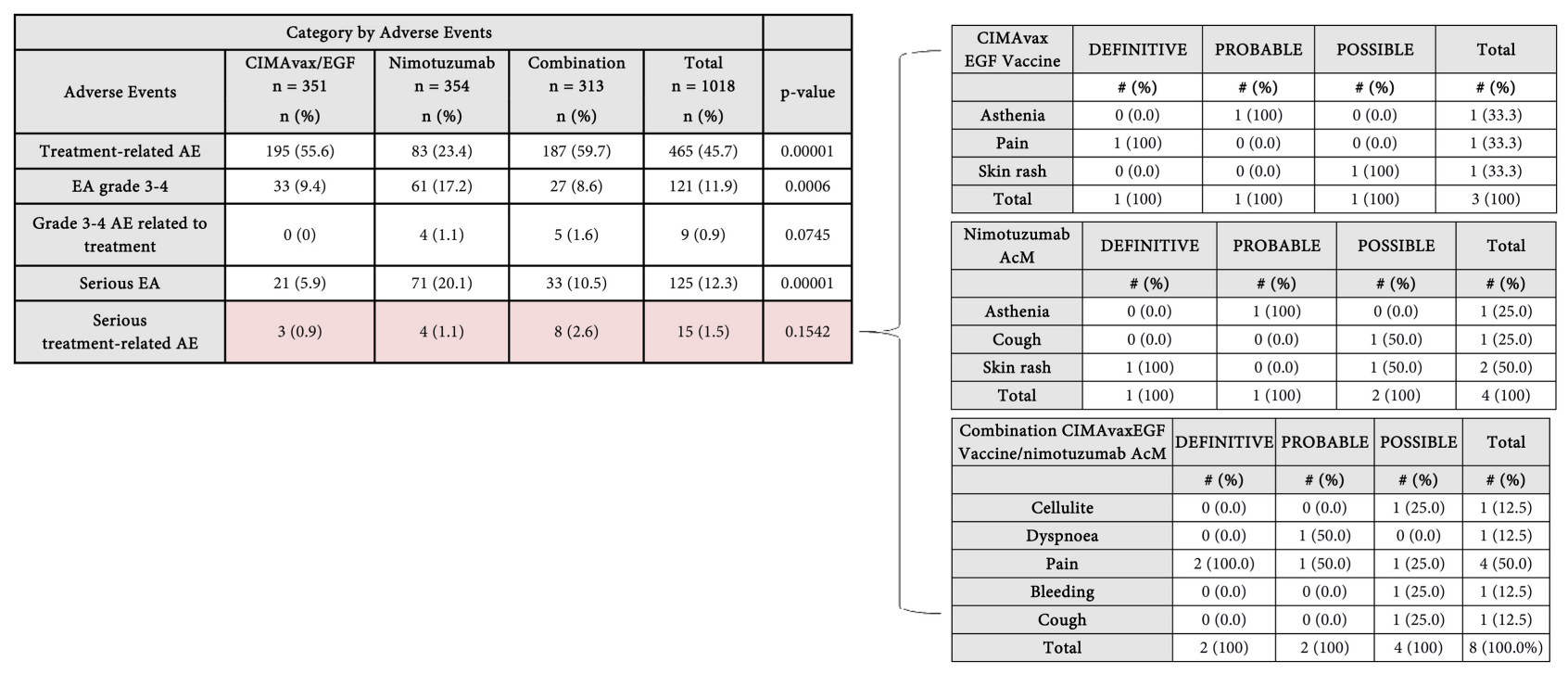

Figure 1. Total AE reported in the expanded clinical use program.

survival was higher than those established for the working hypothesis (three months more than survival reported in 2008, which was 6.2 months) [10]. Figure 2 and Figure 3 describe ITT and PP survival respectively, in it, a trend of benefic can be observed in patients who receiving nimotuzumab (23.55 and 31.14 months respectively).

Although CIMAvax-EGF ${ }^{\circledast}$ vaccine showed numerically lower survival, it was not significant. In this group, a greater number of ADCs from endocervix (32\%) were included, made a difference between the treatment groups $(p=0.049)$. Previously reports showed that this histological variant has a worse response to chemotherapy or radiotherapy treatments in comparison with tumors of epithelial origin variants [14] [15].

Research in cancer treatment has focused on applying treatments that prolong the control of the disease with an adequate quality of life [16] [17]. The survival rate in this study, at 60 months, was greater than $35 \%$ in all treatment groups, unexpected survival for this type of patients (Figure 2 and Figure 3). Since 2014, long-term surviving patients have been reported in the Cuban population treated with CIMAvax-EGF ${ }^{\circledast}$ vaccine in lung cancer [18]. The patients included in this study have a similar behavior to lung cancer refer patients as long term survival (between $37.4 \%-42.7 \%$ for the ITT analysis and 39.7\% - 50\% for PP, Figure 2 and Figure 3) [19].

A therapeutic benefit was observed in patients with ECOG I and previous RT/CT treatment in the group of patients that received the vaccine CIMAVAX-EGF while patients treated with the humanized Mab were benefited when age was over 50 years, ECOG 2 and histological diagnosis of squamous cell carcinoma. These parameters become as a predictive variable, which may influence the appropriate selection of patients to be treated (predictive variables).

Nimotuzumab $\mathrm{mAb}$, whose first therapeutic approach was related to passive 


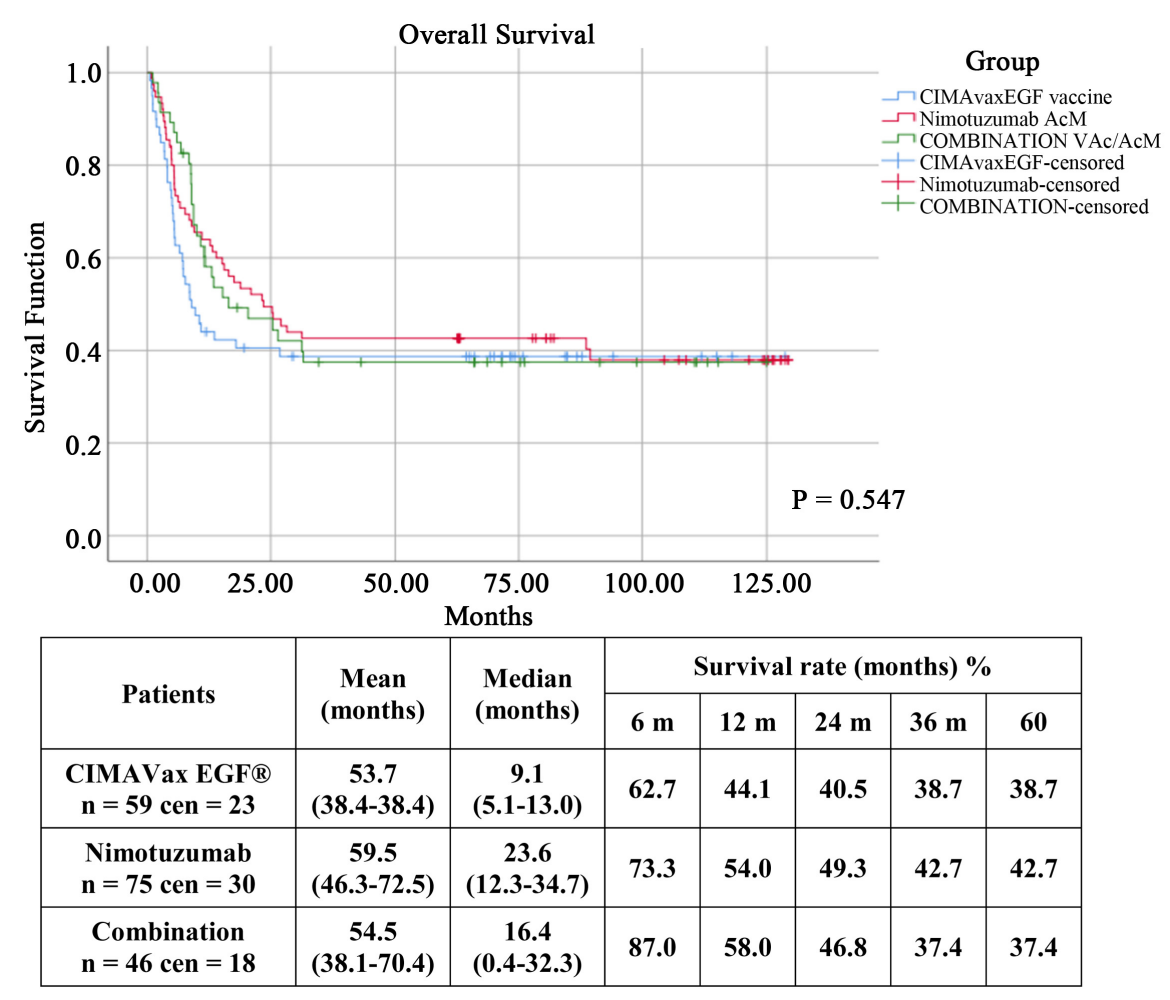

Figure 2. Intent to treat (ITT) overall survival analysis.

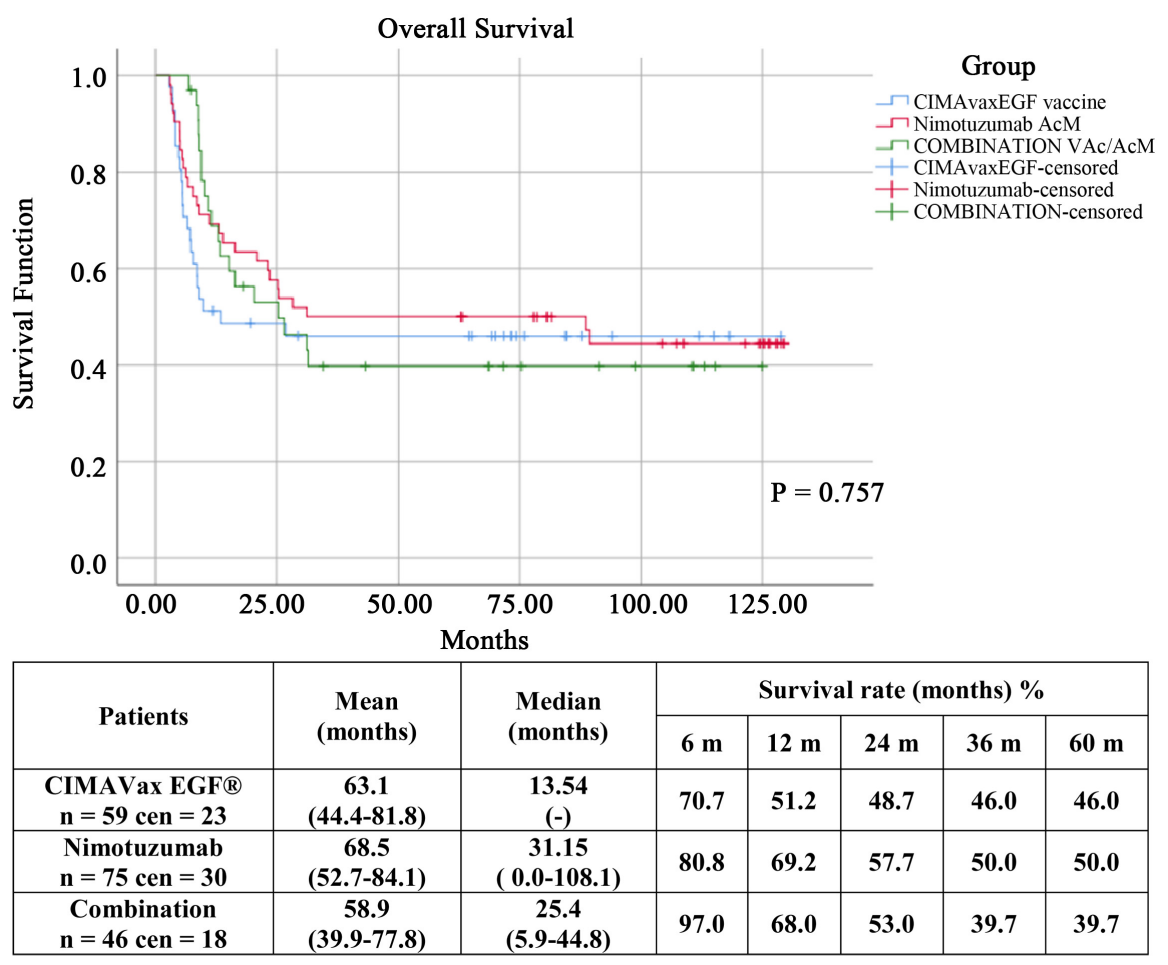

Figure 3. Per Protocol (PP) overall survival analysis.

therapy [12], can directly inhibit tumor growth once it blocks EGF receptor on the cell surface. It inhibits signaling transduction to the nucleus to avoid cell 
proliferation is achieves in less time than the effect of CIMAvax-EGF ${ }^{\circledast}$ vaccine, which requires from two to six weeks to induce antibody response against naive EGF, and thus induce indirectly inhibition of EGFR activation (Figure 4).

Although cervical cancer does not have a high mutation rate in the EGFR signaling chain [20], there are reports of clinical studies that used treatments with intracellular tyrosine kinase inhibitors (TKIs) of the EGFR chain such as sunitinib, imatinib and temsirolimus. These studies do not show evidence of clinical benefit in advanced stages diseases [21]. On the other hand, several authors have reported the use of $\mathrm{mAb}$ against this receptor, such as cetuximab [22], matuzumab [23] and panitumomab [24], with modest results that did not exceed the expectation of the international medical community in comparison with established therapy for this stage of the disease.

A multicenter, open-label, prospective, randomized, phase II clinical trial was conducted in patients with advanced or recurrent cervical cancer, without treatment or after having failed prior chemotherapy $(\mathrm{n}=108)$. In it was comparing carboplatin plus paclitaxel (standard) versus carboplatin plus paclitaxel plus cetuximab therapy and showed that the addition of cetuximab to carboplatin

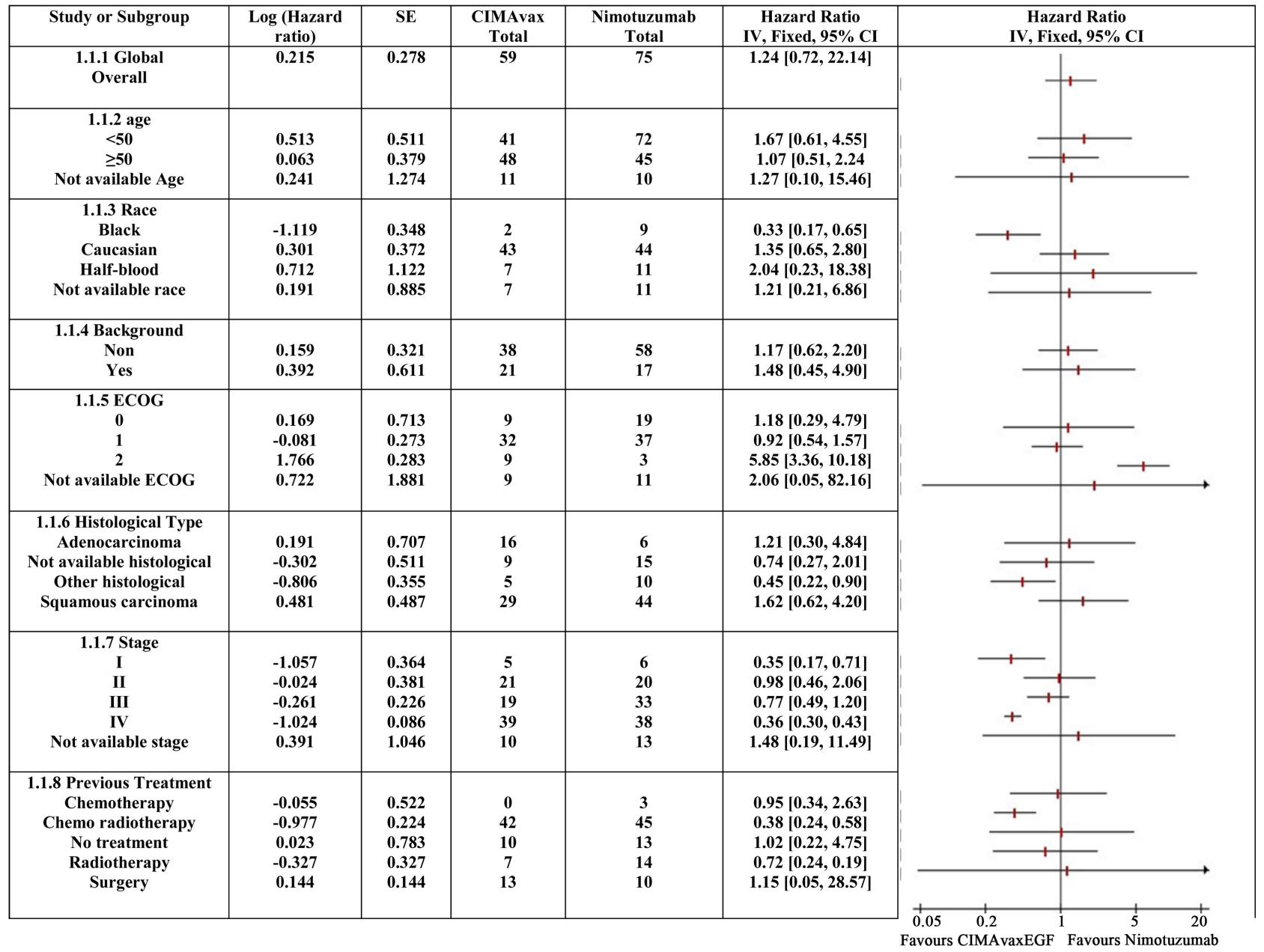

Figure 4. Predictive variables to treatment response for nimotuzumab and CIMAvax-EGF ${ }^{\circledast}$ vaccine groups. 
and paclitaxel were feasible but not more effective than chemotherapy alone. For this reason, cetuximab not proceed to phase III. In this study, chimeric mAb caused grade $\geq 3$ skin toxicities and induced delay in up to two consecutive infusions. The dose reductions were permanent [25]. Current treatment recommendations for persistent, recurrent or metastatic cervical cancer indicate as adequate the carboplatin/paclitaxel or cisplatin/paclitaxel chemotherapy regimen in 253 where the reported survival ranges from 18.3 months to 17.5 months respectively, being more is beneficial in terms of tolerance [26] [27].

The addition of Bevacizumab to this chemotherapy showed an increase in survival and progression-free survival to figures of 17.0 and 8.2 months, respectively, without a deterioration in quality of life despite the adverse reactions reported for this anti VEGFR mAb [28]. Despite being the only mAb reported to date as approved for the indication of cervical cancer in advanced stages, its cost makes it difficult to spread it to all patients who need it, in addition, not all tumors show high expression of this receptor [29].

The increased survival and long-term duration of responses in patients treated with nimotuzumab, after a few doses (at least 6), suggested the blockade of EGFR signaling transduction and the inhibition of tumor cells proliferation, but it was not the only mechanisms of action underlying the efficacy of this antibody. [30] Mazorra et al. demonstrated that sera from patients treated with nimotuzumab, were capable of triggering effector mechanisms such as inhibition of the cell proliferation, ADCC and CDC (in vitro studies), probably due to the presence of specific antibodies against tumor cells promoted for the nimotuzumab action. It is able to trigger the antigenic presentation process that led immunogenic cell death in this assay [31]. We suggest it as a mechanism, which favors the control of the disease and with it, increases the overall survival of the patients.

There is evidence of high expression of HB-EGF and autocrine activation of the same in cervical tumor cells, making it the predominant mechanism of activation of EGFR receptors in this type of tumor [32]. Although EGF expression is associated with macrophages, CCL2 and GM-CSF expression, and macrophage numbers are associated with M-CSFR and CCR2 expression, macrophages do not appear to be the predominant source of HB-EGF activation. Since the relative amount of HB-EGF measured in the epithelial compartment was on average four times higher than the relative amount of HB-EGF in the stromal compartment. These results suggest that, in cervical cancer the main source of tumor cell activation is HB-EGF [32] and for its CIMAVax EGF vaccine is less effective along but in combination with nimotuzumab, any way may become in alternative to treat advanced cervical cancer too.

\section{Conclusion}

CIMAvax-EGF ${ }^{\oplus}$ therapeutic vaccine, nimotuzumab and the combination of both in the treatment of cervical uterine tumors of epithelial origin in advanced stages 
of the disease have an acceptable safety profile and are well tolerated even in prolonged periods. The severe AE with a causal relationship did not exceed 3\% in any of the schemes. The three therapeutic regimens achieved global survival rates at 60 months higher than 35\% in all cases, unprecedented in the literature to date, proposing the design of a phase III clinical trial to consolidate these results using nimotuzumab.

\section{Conflicts of Interest}

The authors declare no conflicts of interest regarding the publication of this paper.

\section{References}

[1] Globocan (2019) The Global Cancer Observatory.

[2] Steller, M.A., Delgado, C.H., Bartels, C.J., Woodworth, C.D. and Zou, Z. (1996) Overexpression of the Insulin-Like Growth Factor-1 Receptor and Autocrine Stimulation in Human Cervical Cancer Cells. Cancer Research, 56, 1761-1765.

[3] Tian, W.J., Huang, M.L., Qin, Q.F., Chen, Q., Fang, K. and Wang, P.L. (2016) Prognostic Impact of Epidermal Growth Factor Receptor Overexpression in $\mathrm{Pa}$ tients with Cervical Cancer: A Meta-Analysis. PLoS ONE, 11, e0158787. https://doi.org/10.1371/journal.pone.0158787

[4] Blanco, R., Cedeño, M., Rengifo, C.E., Blanco, D., Frometa, M., Domínguez, E., et al. (2017) Double Expression of Epidermal Growth Factor Receptor and N-Glycolyl GM3 Ganglioside in Human Malignant Tumors: A Study in Four Different Clinical Scenarios. Advances in Molecular Diagnostics, 2, 112.

[5] Cetina, L., Crombet, T., Jiménez-Lima, R., Ramos-Suzarte, M., Jimenez, L.R., Zapata, S., et al. (2015) A Pilot Study of Nimotuzumab plus Single Agent Chemotherapy Second- or Third-Line Treatment or More in Patients with Recurrent, Persistent or Metastatic Cervical Cancer. Cancer Biology \& Therapy, 16, 684-689. https://doi.org/10.1080/15384047.2015.1026483

[6] Lorente, R.R., Alfonso, S., Sanchez, Y., Viada, C., Ortiz, R., Troche, M., et al. (2016) (2019) Nimotuzumab in Advanced Cervical Cancer: Safety and Efficacy Profile. International Journal of Oncology Research, 2, 16. https://doi.org/10.23937/2643-4563/1710016

[7] Rodriguez, P.C., Popa, X., Martínez, O., Mendoza, S., Santiesteban, E., Crespo, T., et al. (2016) A Phase III Clinical Trial of the Epidermal Growth Factor Vaccine CIMAvax-EGF as Switch Maintenance Therapy in Advanced Non-Small Cell Lung Cancer Patients. Clinical Cancer Research, 22, 3782-3790.

[8] Common Terminology Criteria for Adverse Events V3.0, March 31, 2003. https://ctep.cancer.gov/protocoldevelopment/electronic applications/docs/ctcaev3. pdf

[9] Bain, L.J. and Engelhardt, M. (1991) Statistical Analysis of Reliability and Life-Testing Models. Marcel Dekker, New York.

[10] Monk, J., Still, M., McMeeklin, D.S., et al. (2009) A Randomized Phase III of Four Cisplatin (CIS) Containing Double Combinations in Stage IVB, Recurrent of Persistent Cervical Carcinoma: A Gynecologic Oncology (GOG) Study. Journal of Clinical Oncology, 17, 1-14.

[11] Schrevel, M., Michelle, O.E., Prins, F.A., Baptist, J., Trimbos, M.Z., Fleuren, G.J., et 
al. (2017) Expresión autocrina del factor de crecimiento epidérmico receptor de ligando heparina que se une al factor de crecimiento similar a EGF en cáncer cervical. Revista Internacional de Oncologia, 50, 1947-1954.

[12] Crombet, T., Osorio, M., Cruz, T., Roca, C., del Castillo, R., Mon, R., et al. (2004) Use of the Humanized Anti-Epidermal Growth Factor Receptor Monoclonal Antibody h-R3 in Combination with Radiotherapy in the Treatment of Locally Advanced Head and Neck Cancer Patients. Journal of Clinical Oncology, 22, 1646-1654. https://doi.org/10.1200/JCO.2004.03.089

[13] Saurez, G., Cabanas, R., Zaldívar, M., Garnier, T., Iglesias, B., Piedra, P., et al. (2009) Clinical Experience with Nimotuzumab in Cuban Pediatric Patients with Brain Tumors, 2005 to 2007. MEDICC Review, 11, 27-33. https://doi.org/10.37757/MR2009V11.N3.7

[14] Takeuchi, S. (2016) Biology and Treatment of Cervical Adenocarcinoma. Chinese Journal of Cancer Research, 28, 254-262. https://doi.org/10.21147/j.issn.1000-9604.2016.02.11

[15] Kojima, A., Mikami, Y., Sudo, T., Yamaguchi, S., Kusanagiet, Y., Ito, M., et al. (2007) Gastric Morphology and Immune Phenotype Predict Poor Outcome in $\mathrm{Mu}$ cinous Adenocarcinoma of the Uterine Cervix. The American Journal of Surgical Pathology, 31, 664-672. https://doi.org/10.1097/01.pas.0000213434.91868.b0

[16] Elit, L.M. and Hirte, H. (2014) Management of Advanced or Recurrent Cervical Cancer: Chemotherapy and Beyond. Expert Review of Anticancer Therapy, 14, 319-332. https://doi.org/10.1586/14737140.2014.866041

[17] Schlom, J., Arlen, P.M. and Gulley, J.L. (2007) Cancer Vaccines: Moving beyond Current Paradigms. Clinical Cancer Research, 13, 3776-3782. https://doi.org/10.1158/1078-0432.CCR-07-0588

[18] Sánchez, L., Lorenzo-Luaces, P., Viada, C., Galan, Y., Ballesteros, J., Crombet, T., et al. (2014) Is There a Subgroup of Long-Term Evolution among Patients with Advanced Lung Cancer? Hints from the Analysis of Survival Curves from Cancer Registry Data. Cancer, 14, 933. http://www.biomedcentral.com/1471-2407/14/933 https://doi.org/10.1186/1471-2407-14-933

[19] Lage, A. (2008) Connecting Immunology Research to Public Health: Cuban Biotechnology. Nature Immunology, 9, 109-112. https://doi.org/10.1038/ni0208-109

[20] Ojesina, A.I., Lichtenstein, L. and Freeman, S.S. (2014) Landscape of Genomic Alterations in Cervical Carcinomas. Nature, 506, 371-375. https://doi.org/10.1038/nature12881

[21] Pignata, S., Scambia, G., Lorusso, D., De Giorgi, U., Nicoletto, M.O., Lauria, R., et al. (2019) The MITO CERV-2 Trial: A Randomized Phase II Study of Cetuximab plus Carboplatin and Paclitaxel, in Advanced or Recurrent Cervical Cancer. Gyncologic Oncology, 153, 535-540. https://doi.org/10.1016/j.ygyno.2019.03.260

[22] Santin, A.D., Sill, M.W., Scott, M.D., Leitao, M.M., Jubilee, B., Sutton, G.P., et al. (2011) Phase II Trial of Cetuximab in the Treatment of Persistent or Recurrent Squamous or Non-Squamous Cell Carcinoma of the Cervix: A Gynecologic Oncology Group Study. Gynecologic Oncology, 122, 495-500.

https://doi.org/10.1016/j.ygyno.2011.05.040

[23] Blohmer, J., Gore, M., Kuemmel, S., Alfa, G., et al. (2005) Phase II Study to Determine Response Rate, Pharmacokinetics (PK), Pharmacodynamics (PD), Safety, and Tolerability of Treatment with the Humanized Anti-Epidermal Growth Factor Receptor (EGFR) Monoclonal Antibody EMD 72000 (Matuzumab) in Patients with Recurrent Cervical Cancer. JCO, 23, 2534-2534. 
https://doi.org/10.1200/jco.2005.23.16 suppl.2534

[24] FDA. Lable Information of Vectibix (Panitumumab). http://www.accessdata.fda.gov/drugsatfda docs/label/2009/125147s080lbl.pdf

[25] Moore, K.N., Herzog, T.J., Lewin, S., Giuntoli, R.L., Armstrong, D.K., Rocconi, R.P. and Gold, M.A. (2007) A Comparison of Cisplatin/Paclitaxel and Carboplatin/Paclitaxel in Stage IVB, Recurrent or Persistent Cervical Cancer. Gynecologic Oncology, 105, 299-303. https://doi.org/10.1016/j.ygyno.2006.12.031

[26] Long, H.J., Bundy, B.N., Grendys, E.C., et al. (2005) Randomized Phase III Trial of Cisplatin with or without Topotecan in Carcinoma of the Uterine Cervix: A Gynecologic Group Study. Journal of Clinical Oncology, 23, 2646-4633.

http://www.ncbi.nlm.nih.gov/pubmed/15911865 https://doi.org/10.1200/JCO.2005.10.021

[27] Kitagawar, R., Katsumata, N., Shibata, T., Kamura, T., Kasamatsu, T., Nakanishi, T., et al. (2015) Paclitaxel plus Cisplatin versus Paclitaxel plus Carboplatin in Metastatic or Recurrent Cervical Cancer. The Open-Label Randomized Phase III Trial JCOG 0505. Journal of Clinical Oncology, 33, 2129-2133.

https://doi.org/10.1200//CO.2014.58.4391

[28] Minion, L.E. and Tewari, K.S. (2018) Cervical Cancer-State of the Science: From Angiogenesis Blockade to Checkpoint Inhibition. Gynecologic Oncology, 148, 609-621. https://doi.org/10.1016/j.ygyno.2018.01.009

[29] Bizzarri, N., Ghirardi, V., Alessandri, F. and Venturini, P.L. (2016) Bevacizumab for the Treatment of Cervical Cancer. Expert Opinion on Biological Therapy, 16, 407-419. https://doi.org/10.1517/14712598.2016.1145208

[30] Mazorra, H.Z. and Crombet, R.T. (2014) Pilot Study of a Novel Combination of Two Therapeutic Vaccines in Advanced Non-Small-Cell Lung Cancer Patients. Cancer Immunology, Immunotherapy, 63, 737-747. https://doi.org/10.1007/s00262-014-1552-9

[31] Mazorra, Z., Lavastida, A., Concha-Benavente, F., Valdés, A., Srivastava, R.M., García-Bates, T.M., et al. (2017) Nimotuzumab Induces NK Cell Activation, Cytotoxicity, Dendritic Cell Maturation and Expansion of EGFR-Specific T Cells in Head and Neck Cancer Patients. Frontiers in Pharmacology, 8, 382.

http://www.frontiersin.org https://doi.org/10.3389/fphar.2017.00382

[32] Schrevel, M., Osse, E.M., Prins, F.A., et al. (2017) Autocrine Expression of the Epidermal Growth Factor Receptor Ligand Heparin-Binding EGF-Like Growth Factor in Cervical Cancer. International Journal of Oncology, 50, 1947-1954.

https://doi.org/10.3892/ijo.2017.3980 